\title{
Extract and Classification of Iris Images by Fractal Dimension and Efficient Color of Iris
}

\author{
Mahdi Jampour \\ Computer and IT Department at \\ Kerman Institute of Higher Education (KIHE) \\ Kerman, Iran \\ Majid Estilayee \\ Computer and IT Department at \\ University of Apply Science and Technology \\ Zarand, Iran
}

\author{
Ali Naserasadi \\ Industrial and Mining Faculty at \\ Shahid Bahonar University of Kerman \\ Kerman, Iran
}

\author{
Maryam Ashourzadeh \\ Computer and IT Department at \\ Kerman Institute of Higher Education (KIHE) \\ Kerman, Iran
}

\begin{abstract}
In the last decade, identification by biometric features such as iris and fingerprint has been considered very much. Last introduced methods, in fact, could achieve high accuracy, but one of the most common problems in these methods is the lack of scalability. So these methods are suitable for use in small databases of iris. One solution for this problem is using the hierarchy classification. In this paper, fractal dimension of iris and effective range of color in RGB layers are used as first and second layers of classification in iris images respectively in order to increase the performance of different methods in human identification. The result of simulation on Phoenix database's data shows that this method is suitably efficient in the classification step.
\end{abstract}

\section{Keywords}

Iris Classification, Fractal Theory, Fractal Dimension.

\section{INTRODUCTION}

Biometric term is derived from two ancient Greek words: Bios that means life and Metron that means measure. This term refers to the methods of human identification based on one or more physiological or behavioral characters of a person. Biometric is the science of human identification through innate characteristics such as fingerprint, palm, face, signature, handwriting, iris and retina scan, voice and etc. In biometric systems, identification and authentication processes use innate characteristics instead of artificial ones such as passwords and etc, which causes increasing of security in these processes. Biometric characteristics can be divided in to three groups:

- Physical characteristics

- Behavioral characteristics

- Chemical characteristics

\subsection{Physical Characteristics}

This feature analyses and recognizes of one physical characteristic such as fingerprint or hand geometry. Organ measurement is one of the oldest methods of human identification which has been diversified with the development of technology. Identification methods via iris face or retina veins are the other ways in this case.

\subsection{Behavioral Characteristics}

Behavioral techniques measure the way of doing a special work by human as like as signing or saying a phrase. The methods of this group are identification via signature, typing rhythm, voice and etc.

\subsection{Chemical Characteristics}

These techniques measure a chemical feature of user. Some of these methods are identification via DNA, blood sugar, body odor and etc.

A quick-look comparison of all of the biometric technologies is provided in Table 1. In this comparison the biometric technologies have been compared on the following four criteria: mechanism simplicity, accuracy, integrity and cost [1]. It is obvious that the iris and fingerprint features are more appropriate than other biometric features. In addition, the iris has many other properties which make it distinguished. For example, it is the only internal body member which is visible from out and protected from environment nicely. Capturing iris image is contact less which is the most important parameter of user unsatisfactory. Also, it forms from first year of life and remain unchangeable up to the end of life. In addition, iris structure is full of Physiometric information, which means there exist more than 200 analyzable properties inside iris and they cannot be duplicated like as fingerprint.

Table 1: The comparison of human identification methods includes biometric and non-biometric features.

\begin{tabular}{|l|c|c|c|c|}
\hline $\begin{array}{c}\text { Biometric } \\
\text { Features }\end{array}$ & $\begin{array}{c}\text { Mechanism } \\
\text { Simplicity }\end{array}$ & Accuracy & Integrity & Cost \\
\hline Fingerprint & 7 & 7 & 4 & 3 \\
\hline Signature & 3 & 4 & 5 & 4 \\
\hline
\end{tabular}




\begin{tabular}{|l|c|c|c|c|}
\hline Facial geometry & $\mathbf{9}$ & $\mathbf{4}$ & $\mathbf{7}$ & $\mathbf{5}$ \\
\hline Eye iris & $\mathbf{8}$ & $\mathbf{9}$ & $\mathbf{8}$ & $\mathbf{8}$ \\
\hline Eye retina & $\mathbf{6}$ & $\mathbf{8}$ & $\mathbf{5}$ & $\mathbf{7}$ \\
\hline Hand geometry & $\mathbf{6}$ & $\mathbf{5}$ & $\mathbf{6}$ & $\mathbf{5}$ \\
\hline Finger geometry & $\mathbf{7}$ & $\mathbf{3}$ & $\mathbf{7}$ & $\mathbf{4}$ \\
\hline Hand veins & $\mathbf{6}$ & $\mathbf{6}$ & $\mathbf{6}$ & $\mathbf{5}$ \\
\hline Ear anatomy & $\mathbf{5}$ & $\mathbf{4}$ & $\mathbf{7}$ & $\mathbf{5}$ \\
\hline Voice & $\mathbf{4}$ & $\mathbf{2}$ & $\mathbf{3}$ & $\mathbf{2}$ \\
\hline DNA & $\mathbf{1}$ & $\mathbf{7}$ & $\mathbf{9}$ & $\mathbf{9}$ \\
\hline Odor & $\mathbf{2}$ & $\mathbf{2}$ & $\mathbf{7}$ & $\mathbf{?}$ \\
\hline Key stroke & $\mathbf{4}$ & $\mathbf{1}$ & $\mathbf{2}$ & $\mathbf{1}$ \\
\hline $\begin{array}{l}\text { Password and } \\
\text { Comparison }\end{array}$ & $\mathbf{5}$ & $\mathbf{2}$ & $\mathbf{8}$ & $\mathbf{1}$ \\
\hline
\end{tabular}

- fractional dimension

There are many instances of fractals in nature. For example, Contour set, Sierpinski triangle, Koch snow, Julia set, Fern fractal, etc. Figure 1 shows some fractals.
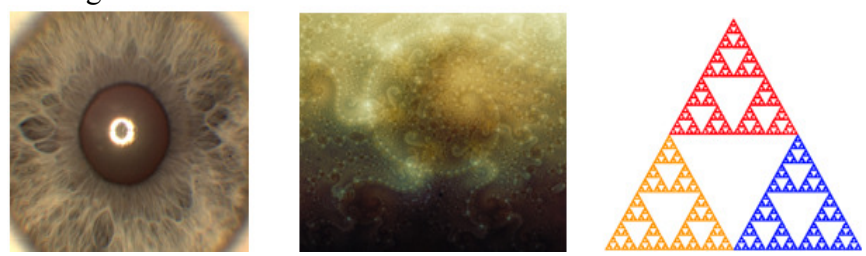

Figure1. Some instances of fractal images

\subsection{Fractal Dimension}

The most important property of fractals is their decimal dimension. We should remember that a line has 1 dimension, a surface has 2 dimensions and a cube has 3 dimensions. Dimension is an independent term from size. There are many methods for calculating fractal dimension. Some of the most famous are:

1- Hausdorff dimension

2- Packing dimension

3- Rényi dimensions

4- Box-Counting dimension

5- Correlation dimension

In 1993, John Daugman presented the first iris identification system by Gabor wavelet transform. Daugman idea was based on transforming iris image to a 256 Byte code that is named Iris Code. Then, by comparison of iris codes distance, as like as Hamming distance, he measured similarity between two irises [2, 3]. In 1996, Wildes suggested the automatic iris identification system that was a system with high computational complexity $[4,5]$. Wageeh was another researcher in this context. System that he presented uses wavelet transformation in different levels. It, also, uses the one dimensional resulted signal for comparison with basic instance [6]. Zhu, Tan and Sanchez also presented other techniques [7, 8, and 9]. It can be said that most of old techniques were based on multiscale analyzes.

In recent years, new techniques based of fractal are presented according to fractal properties of iris and other features [10, 11]. These techniques are very stable in small databases, so most of them are restricted to databases with about 1000 images. Systems in developed enterprises in all over the world also have above constraint. International centers, as like as airports that some countries have started to use automatic iris identification systems in them, also have many constraints. In the most of automatic iris identification systems, normally, after extracting one of iris properties, the comparison and identification processes are started directly, which cause some restrictions in identification. So, it is participating that combining more parameters can improve results and increase instance images domain.

\section{Fractal Theory}

The Fractal Theory was given by Benoit Mandelbrot in 1975 $[12,13]$. In his opinion, fractal objects have 3 important properties.

- $\quad$ self similarity

- $\quad$ iterative formation
For example, in fractal dimension calculating by Box-Counting dimension method, we divide the space into some boxes with size of $\varepsilon$, and count boxes that the fractal has effect on them. Then we can calculate the fractal dimension via relation (1). The result essentially is a decimal number.

$$
\operatorname{Dim}_{B o x}(S)=\lim _{\varepsilon \rightarrow 0} \frac{\log N(\varepsilon)}{\log (1 / \varepsilon)}
$$

\section{HybRId ClassificATION OF IRIS IMAGES}

At all, hybrid classification of iris images is done via two effective parameters: fractal dimension and effective color of iris extraction. Both of these parameters have suitable complexity that increases the quality of classification of iris images algorithm. First step in this process is calculating of fractal dimension. The next step is extracting the iris color.

\subsection{Extracting Iris}

The first step in iris classification is extracting iris from image and transform the iris image into a unique and standard image. To achieve this goal, we extract the iris from image via image histogram and then by a geometrical rotation transformation, we map its circular shape into a rectangular one. This transform has been shown in relation (2). The result is iris extraction with all of its partial information. Of course, this process does not have any negative side effect on color based analysis that will take place in next step. Figure 2, shows some analyzed images with extracted irises. As you see, the result of this step is some same rectangles that are very suitable for fractal dimension analysis. 


$$
\begin{aligned}
& X r o t=x_{1} \times \operatorname{Cos}(\mathrm{deg})+y_{1} \times \operatorname{Sin}(\mathrm{deg}) \\
& Y r o t=y_{1} \times \operatorname{Cos}(\mathrm{deg})-x_{1} \times \operatorname{Sin}(\mathrm{deg})
\end{aligned}
$$
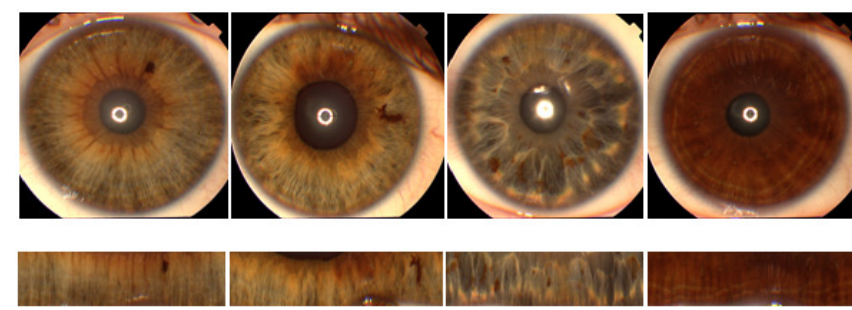

Figure2. Some iris image with extracted spectrum

\subsection{Calculating the Fractal Dimension}

For calculating fractal dimension, we use Box-Counting method that relation (1) shows its process [14, 15]. Calculating fractal dimension of Phoenix database instances showed that the fractal dimension range was [1.30 1.90]. By classification of this range into four groups, following domain for each group are obtained. Table 2 shows the result of using the above algorithm on Phoenix database data.

$$
\begin{aligned}
& \text { Group A }=[1.30 \sim 1.45] \\
& \text { Group B }=[1.45 \sim 1.60] \\
& \text { Group C }=[1.60 \sim 1.75] \\
& \text { Group D }=[1.75 \sim 1.90]
\end{aligned}
$$

\subsection{Extracting Effective Color from Iris Images}

Iris structure has unique properties and a complex pattern. Iris color is one of them. Iris color and pattern creation is based on Melanin Pigments and Crypts counts. Human iris color has a wide range, so that we can use these different colors for documentation [16]. This range is changing from dark brown to light blue [17]. In classification of different iris colors, we can use some terms such as brown, honey eye, green, gray, blue or a combination of them. But because of lack of an explicit border between these colors, we classified them into three classes: light, dark and average and then, calculated the average of color value in each layer of RGB levels. In other word, we calculated the average of red, green and blue color value in each image. Also, because of ambiguity in light, average and dark terms, we calculated the value of each red, green and blue layer via experience and used these values for classification. These parameters are as following for different images.
1. Light Iris: value of $R$ should be greater than 105 , value of $G$ should be greater of 90 and value of $B$ should be greater than 40.

2. Dark Iris: value of $R$ should be smaller than 110 , value of $G$ should be smaller of 70 and value of $B$ should be smaller than 50 .

3. Average Iris: the iris that does not belong to two previous classes.

Table 3 shows the results of classification all of database images via the above algorithm. These results are fully matching with reality according to iris color.

\section{IMPLEMENTATION AND RESULTS}

Combining the two ideas that presented at previous section, give the ability of classifying of many iris image instances into groups with suitable frequency (almost uniform) to the algorithm. One of the most important attributes of this algorithm is the uniformity of frequency in each group. The time complexity of algorithm is very suitable, too. The above technique was implemented in MATLAB environment in a P4 computer that was equipped with Windows XP operating system. The sample database has 4096 instances. The final results of each classified group with this algorithm are showed at Table 4 in 2 classification layers.

\section{Conclusion}

Iris Identification is one of the most reliable methods for human identification. Iris's features have many details so they can be used for human classification and identification. Combining iris's features can increase the iris classification stability. On the other hand, iris is a fractal phenomenon, and has fractals features such as fractional dimension; so combining this structure with extracted color of iris can classify a wide range of iris samples. In this paper we used this method. The results of combining these two properties increased the performance of the iris classification system. So, it can improve identification process's time complexity. Focus on extraction of iris's features can result very fast identification systems in the future. Because of using of pattern recognition processes in existing systems, most of them don't have suitable time complexity. By using hybrid classification of iris's features and then searching in each class, time complexity decrease in a nice manner. 
Table2. Fractal dimension based image classification

\begin{tabular}{|c|c|c|c|c|c|}
\hline Class & A & B & C & D & Sum \\
\hline Frequency & 72 & 84 & 156 & 72 & 384 \\
\hline
\end{tabular}

Table3. Iris image classification base on color

\begin{tabular}{|c|c|c|c|c|}
\hline Class & Light & Average & Dark & Sum \\
\hline Frequency & 132 & 144 & 108 & 384 \\
\hline
\end{tabular}

Table4. The results of presented algorithm running on Phoenix iris images database

\begin{tabular}{|c|c|c|c|c|c|c|c|c|c|c|c|c|}
\hline $\begin{array}{c}\text { Class Based } \\
\text { On } \\
\text { Dimension }\end{array}$ & \multicolumn{3}{|c|}{ Class A } & \multicolumn{3}{|c|}{ Class B } & \multicolumn{3}{|c|}{ Class C } & \multicolumn{3}{|c|}{ Class D } \\
\hline Frequency & \multicolumn{3}{|c|}{768} & \multicolumn{3}{|c|}{896} & \multicolumn{3}{|c|}{1664} & \multicolumn{3}{|c|}{768} \\
\hline $\begin{array}{l}\text { Class based } \\
\text { on color }\end{array}$ & Light & Average & Dark & Light & Average & Dark & Light & Average & Dark & Light & Average & Dark \\
\hline Frequency & 128 & 128 & 512 & 320 & 256 & 320 & 640 & 704 & 320 & 320 & 384 & 64 \\
\hline
\end{tabular}

\section{REFERENCES}

[1] Jain, A.K. Ross, A. Prabhakar, S. "An introduction to biometric recognition." IEEE Trans. Circuits and Systems for Video Technology vol. 14, no. 1, Jan. 2004.

[2] John Daugman. "High confidence visual recognition of persons by a test of statistical independence." IEEE Transactions on Pattern Analysis and Machine Intelligence, Vol. 15, no. 11, pp.1148-1161, Nov. 1993.

[3] John Daugman. "How iris recognition works." IEEE Transactions on Circuits and Systems for Video Technology, Vol. 14, no. 1, pp.21-30. 2004.

[4] Richard P. Wildes. "Iris recognition: An emerging biometric technology." Proceedings of the IEEE, Vol. 85, no. 9, pp. 1348-1363, Sept. 1997.

[5] Richard P. Wildes, Jane C. Asmuth, Gilbert L. Green, et al. "A system for automated iris recognition." Second IEEE Workshop on Applications of Computer Vision, pp. 121-128. 1994.

[6] Wageeh Boles and Boualem Boashash. "A human identification technique using images of the iris and wavelet transform." IEEE Transactions Signal Processing, Vol. 46, no. 4, pp. 1185-1188, April. 1998.

[7] D. de Martin-Roche, Carmen Sanchez-Avila, and Raul Sanchez-Reillo. "Iris recognition for biometric identification using dyadic wavelet transform zero- crossing." In IEEE Int. Carnahan Conf. on Security Technology, pp. 272-277. 2001.

[8] Judith Liu-Jimenez, Raul Sanchez-Reillo, and Carmen Sanchez-Avila. "Biometric co-processor for an authentication system using iris biometrics." IEEE Int. Conf. Security Technology, pp. 131-135. 2004.
[9] Li Ma, Tieniu Tan, Yunhong Wang, and Dexin Zhang. "Personal identification based on iris texture analysis." IEEE Transactions on Pattern Analysis and Machine Intelligence, 25(12):1519-1533. 2003.

[10] Jampour, M. Shojaei, H. Ashourzadeh, M. Yaghoobi, M. "Compressing of Fingerprint Images by Means of Fractals Feature.” IEEE Int. Conf. ICMV’09. pp. 18-22. 2009.

[11] Jampour, M. Yaghoobi, M. Ashourzadeh, M. Soleimani, A. "A New Fast Technique for Fingerprint Identification with Fractal and Chaos Game Theory" Fractals. Vol. 18, Issue 3, pp. 293-300. 2010.

[12] Mandelbrot, B. Fractals: Form, Chance, and Dimension. W. H. Freeman and Co, San Francisco. 1977.

[13] Mandelbrot, B. "A multifractal walk down Wall Street." SCI AMERICAN INC. vol. 280, no. 2, Pp. 70-73. 1999.

[14] Barnsley, M. F. Fractals Everywhere. Academic Press, New York, 1988.

[15] Barnsley, M. F. SuperFractals. Cambridge University Press, New York, 2006.

[16] German EJ, Hurst MA, Wood D, Gilchrist J. "A novel system for the objective classification of iris color and its correlation with response to $1 \%$ tropic amide." Ophthalmic Physiol Opt. 1998.

[17] Huiqiong Wang, Stephen Lin, Xiaopei Liu, Sing Bing Kang. "Separating Reflections in Human Iris Images for Illumination Estimation." Proc. IEEE Int. Conf. Computer Vision, 2005.

[18] Jampour, M. Yaghobi, M. Ashourzadeh, M. "Fractal Images Compressing by Estimating the Closest Neighborhood with Assistance of Schema Theory." J. Com. Sci. Vol. 6, no. 5, pp. 591-596. 2010. 